dine nucleotide and flavine nucleotide readily with the aid of molecular hydrogen.

The same preparation formed butyrate from crotonate utilizing hydrogen gas as reducing agents. This reduction proceeded quantitatively by the addition of acetyl phosphate (or acetyl-CoA) and 2,3-dimercaptopropanol (BAL) at $\mathrm{pH} \mathrm{7.2,} \mathrm{while} \mathrm{it} \mathrm{was} \mathrm{severely} \mathrm{in-}$ hibited by arsenate or acetate. It was pointed out that the reduction of crotonate was accelerated by FAD to some extent.

The mechanism of the reduction of crotonate to butyrate under atmospheric hydrogen was considered to involve the activation of both crotonate and molecular hydrogen, and the reduction of crotonyl-CoA to butyryl-CoA.

[Agr. Biol. Chem., Vol. 25, No. 4, p. 313 318, 1961]

\title{
Effect of Gamma-Ray upon Food Microorganisms
}

Part VIII. Influence of the Addition of some Preservatives or Antibiotics

\author{
By Wataru Watanabe
}

Department of Agricultural Chemistry, Tokyo University of Education and the Nikô Institute of Food, Nikô Co., Ltd.

Received September 13, 1960

\begin{abstract}
Possibility of enhancement in the lethal effect of gamma-ray by the aid of various substances was investigated. This report deals with the survival-ratios of four strains of bacteria in the nutrient or the non-nutritious pure agar medium, to which various kinds of preservatives or antibiotics were added.
\end{abstract}

\section{INTRODUCTION}

The foodstuffs irradiated with gamma-ray can be preserved for a longer period owing to the decreased number of microorganisms in them. However, it is often observed that many foodstuffs undergo undesirable change in quality by the irradiation. Therefore, it is necessary to investigate methods that enhance the lethal effect of gamma-ray, by which the foodstuffs can be preserved by means of irradiation at a dose causing no such undesirable change.

The author has conducted a series of studies concerning the environmental conditions be- fore, during or after gamma-ray irradiation affecting the survival of bacteria, and has reported that none of them enhanced the lethal effect of gamma-ray in nutritious state $e^{1 \sim 7)}$.

Heat processing or the addition of antibiotics or various preservatives are some of such methods. Shewan has conducted studies concerning the preservation of fish meat by a combined application of antibiotics and gam-

\footnotetext{
1) W. Watanabe, This Journal, 22, 68 (1958).

2) W. Watanabe, This Journal, 22, 255 (1958).

3) W. Watanabe, This Journal, 23, 73 (1958).

4) W. Watanabe, This Journal. 24, 75 (1960).

5) W. Watanabe, This Journal. 24, 84 (1960).

6) W. Watanabe, This Journal. 24, 673 (1960)

7) W. Watanabe, This Journal. 24, 681 (1960).
} 
ma-ray irradiation and reported that the codfish or mackerel fish meat containing tetracycline could be preserved for a little longer than the control's). Akimoto has examined the effect of the addition of streptomycine, chloramphenicol or penicillin upon the gamma-ray irradiation of the pure cultures of various microorganisms in a non-nutritious medium and reported a slightly enhanced effect on the survival of the bacteria9).

However, no method has been established that enhances the lethal effect of gamma-ray. The author has, therefore, carried out studies in line with this point, but none of the additives tested enhanced the lethal effect of gamma-ray as presented below.

\section{EXPERIMENTAL}

The bacteria tested were $E$. coli, A. aerogenes, Prot. vulgaris and Pseud. aeruginosa. The preservatives tested were sodium benzoate, DHA (dehydroacetic acid), and Z-furan (5-nitro-2-furylacrylic amide, $10 \%$ pure), and the antibiotics Achromycin (tetracycline) and Terramycin (oxytetracycline). In order to carry out the experiments by the simplified method, the maximum tolerating dose of each substance was determined with each species of bacteria. Then, one tenth concentration of the maximum tolerating dose was applied in the experiment.

The used media were the nutrient agar medium and the pure agar medium. The radiation source was cobalt 60 of 50 curie, placed at School of Medicine, Keio University. Six Petri dishes were irradiated at room temperature within a range of $18^{\circ} \mathrm{C}$ to $20^{\circ} \mathrm{C}$. The duration of irradiation was 2 hours. The bacterial concentration of a sample subjected to irradiation was $4.0-9.9 \times 10^{2}$ cells $/ \mathrm{ml}$. Antibiotics were dissolved in saline at the concentration of $1,000 \mathrm{mcg} / \mathrm{ml}$, which were stored in a deep-freezer. These stock solutions were thawed, diluted, and one tenth volume was added to the medium to give a desired final concentration. The detail of experimental procedures will be mentioned in the text.

\section{RESULTS}

\section{(1) Addition of Sodium Benzoate}

The maximum tolerating doses differed

\footnotetext{
8) J.M. Shewan and J. Liston, Peaceful Uses of Atomic Energy, 27, 377 (1958).

9) T. Akimoto, Jap. Radio-isotopes, 9, 6 (1960) (in Jap.).
}

from one species to another as was the case with thiourea. In the nutrient agar medium, the concentrations of this agent tested were $1: 5 \times 10^{2}$ and $1: 1 \times 10^{3}$ with E. coli, $1: 2 \times 10^{3}$ with $A$. aerogenes, $1: 5 \times 10^{3}$ with Prot. vulgaris and $1: 1 \times 10^{3}$ with Pseud. aeruginosa. All the survival curves are diagrammed in Figure 1 5, which indicates that the addition of this agent resulted in the protection of the four species of bacteria tested from the lethal effect of gamma-ray.

In the pure agar medium, the concentrations of sodium benzoate added were $1: 1 \times 10^{4}$

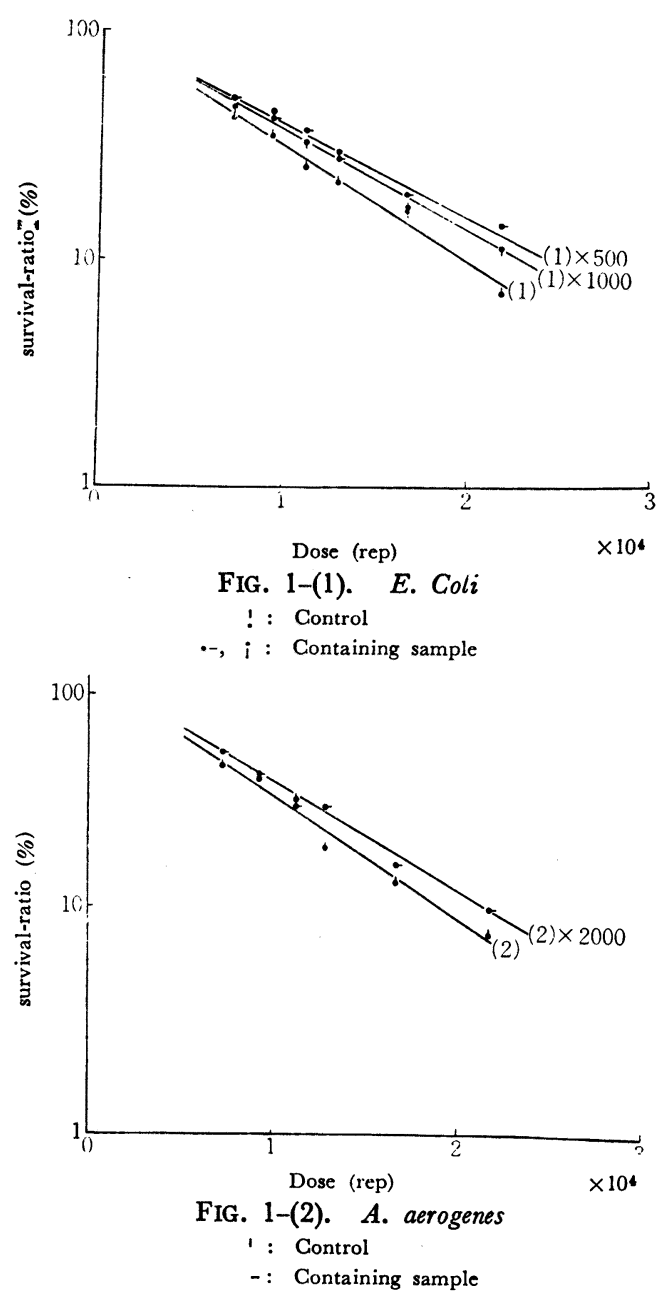




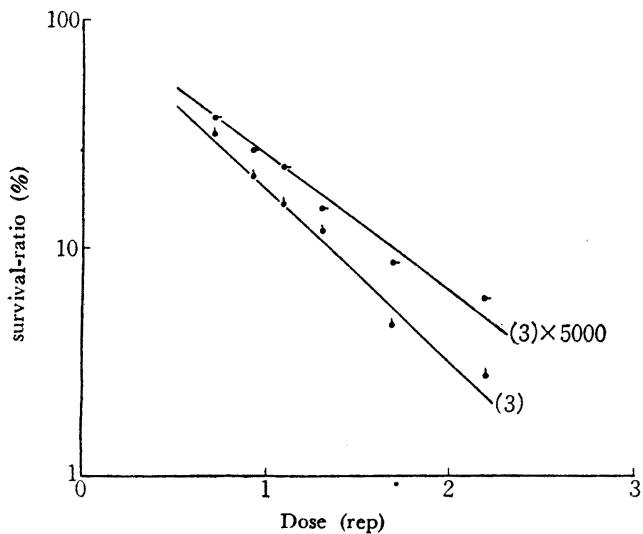

Fig. 1-(3). Prot. vulgaris

!: Control --: Containing sample

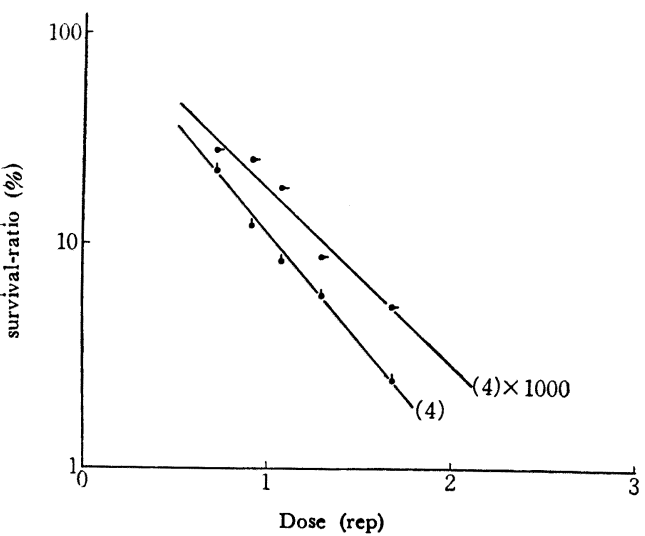

F1G. 1-(4). Pseud. aeruginosa.

!: Control .-: Containing sample

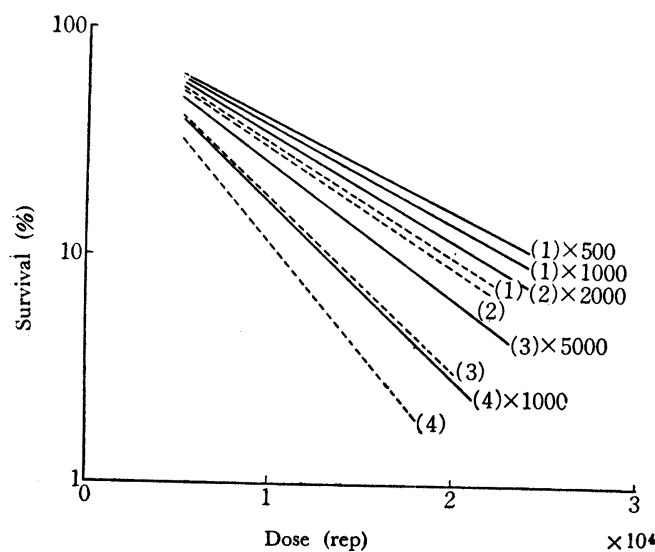

FIG. 1-(5). Survival-curves of Bacteria Irradiated in nutrient media containing sodium benzoate.

(1) E. coli (2) A. aero. (3) Pr. vul. (4) Ps. aerug. with E. coli, $1: 2 \times 10^{4}$ and $1: 5 \times 10^{4}$ with $A$. aerogenes, $1: 1 \times 10^{5}$ with Prot. vulgaris and $1: 1 \times 10^{4}$ with Pseud. aeruginosa. After irradiation, all samples were covered with the nutrient agar prior to incubation. The results are diagrammed in Figure 2, which shows a lower survival ratio in every case, as was the case with thiourea in the pure agar medium.

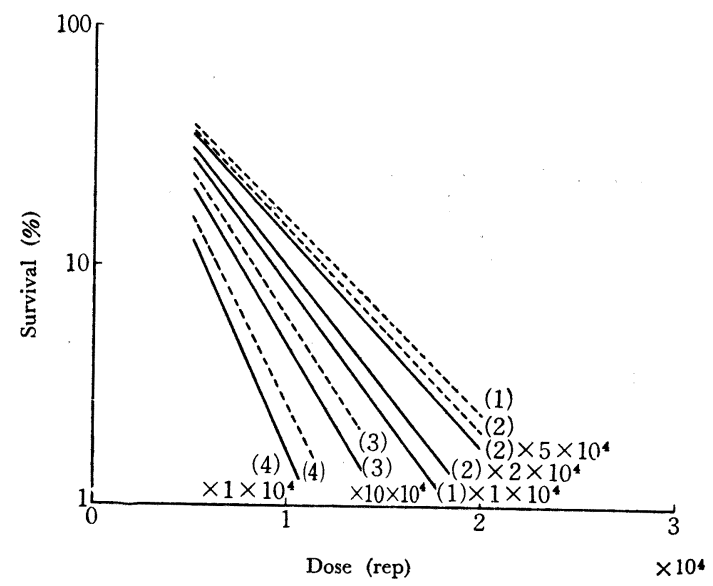

Fig. 2. Survival-curves of Bacteria Irradiated in Pure Agar Media Containing Sodium Benzoate.

(1) E. coli (2) A. aero. (3) Pr. vul. (4) Ps. aerug. ....... control

\section{(2) Addition of DHA}

The concentrations tested in the nutrient agar medium were $1: 5 \times 10^{2}$ and $1: 1 \times 10^{3}$ with $E$. coli, $1: 1 \times 10^{3}$ with $A$. aerogenes, 1: $5 \times 10^{3}$ with Prot. vulgaris and $1: 1 \times 10^{3}$ with Pseud. aeruginosa. The results shown in Figure 3 give more or less higher survival ratios with DHA.

In the pure agar medium, the concentrations of DHA tested were $1: 2 \times 10^{5}$ with both $E$. coli and $A$. acrogenes, $1: 1 \times 10^{5}$ with Prot. vulgaris and $1: 2 \Varangle_{10} 10^{5}$ with Pseud. aeruginosa. All the survival curves are diagrammed in Figure 4. As can be seen in the figure, the addition of DHA slightly decreased the survival ratios of the organisms. 


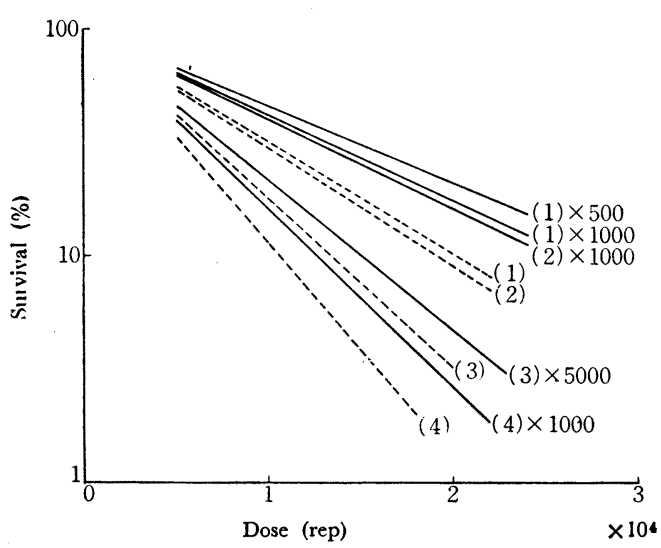

FIG. 3. Survival-curves of Bacteria Irradiated in Nutrient Media Containing DHA.

(1) E. coli (2) A. aero. (3) Pr. oug. (4) Ps. aerug.

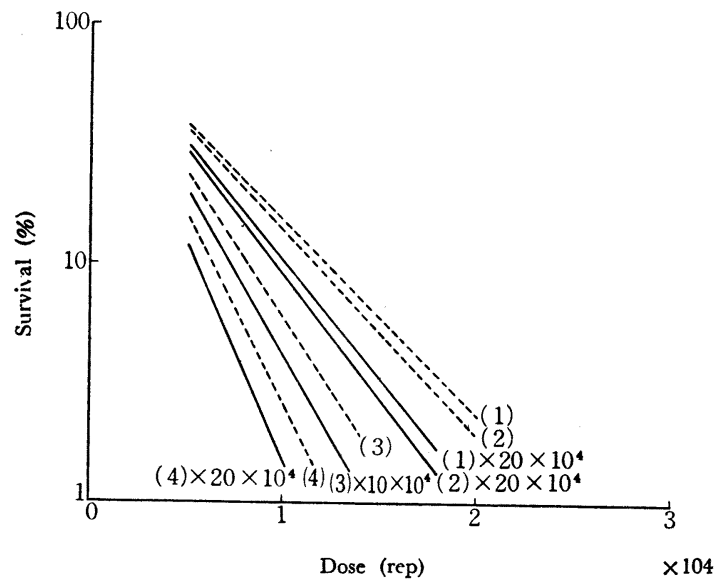

FIG. 4. Survival-curves of Bacteria Irradiated in Pure Agar Media Containing DHA.

(1) E. coli (2) A. aero. (3) Pr. vul. (4) Ps. aerug.

\section{(3) Addition of Z-furan}

The concentrations of Z-furan added to the nutrient agar medium were $1: 5 \times 10^{2}$ and $1: 1 \times 10^{3}$ with $E$. coli, $1: 3 \times 10^{5}$ with $A$. aerogenes, and $1: 2 \times 10^{5}$ with Prot. vulgaris and Pseud. aeruginosa. All the survival curves are diagrammed in Figure 5. As was in the previous experiments, protective effect was observed.

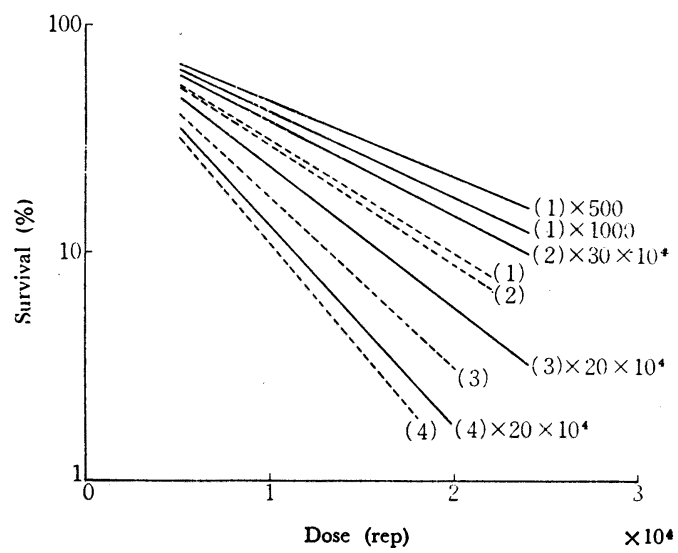

FIG. 5. Survival-curves of Bacteria Irradiated in Nutrient Media Containing 2-Furan.

(1) E. coli (2) A. aero. (3) Pr. vul. (4) Ps. aerug. -...-.. cortrol

The concentrations of this agent added to the pure agar medium were $1: 2 \times 10^{5}$ with E. coli, $1: 3 \times 10^{5}$ with $A$. aerogenes, $1: 2 \times 10^{6}$ with Prot. vulgaris and Pseud. aeruginosa. The results are shown in Figure 6, indicating lower survival ratios of the strains than the controls.

\section{(4) Addition of Achromycin}

The concentration of the antibiotic added

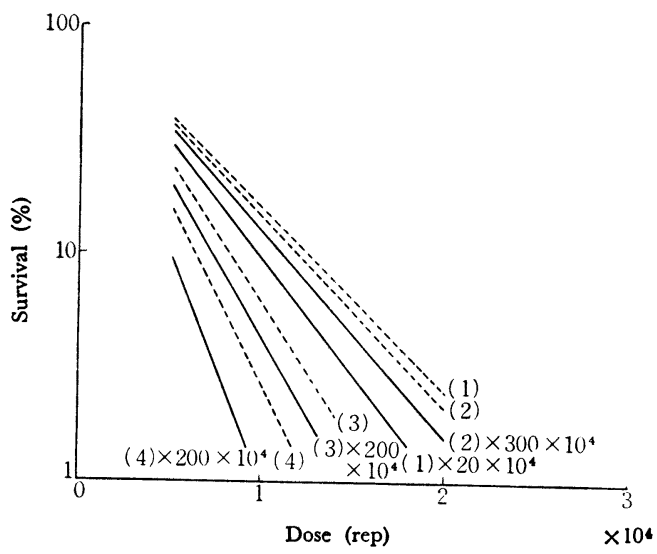

FIG. 6. Survival-curves of Bacteria Irradiated in Pure Agar Media Containing Z-furan.

(1) E. coli (2) A. aero. (3) Pr. vul. (4) Ps. aerug. 
to the nutrient agar medium was $1 \mathrm{mcg} / \mathrm{ml}$ for $E$. coli, $A$. aerogenes, or Pseud. aeruginosa and for Prot. vulgaris $0.1 \mathrm{mcg} / \mathrm{ml}(\gamma)$ was added. The survival curves obtained are diagrammed in Figure 7. As can be seen, it was found that the addition of Achromycin resulted in higher survival ratios of these organisms, indicating similar protective effect as was the case with preservatives.

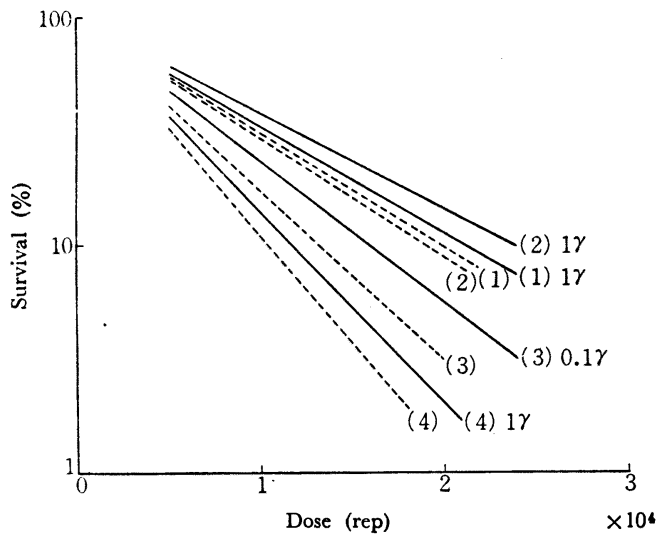

FIG. 7. Survival-curves of Bacteria Irradiated in Nutrient Media Containing Achromycin. (1) E. coli (2) A. aero. (3) Pr. vul. (4) Ps. aerug. -.-.-.-: control

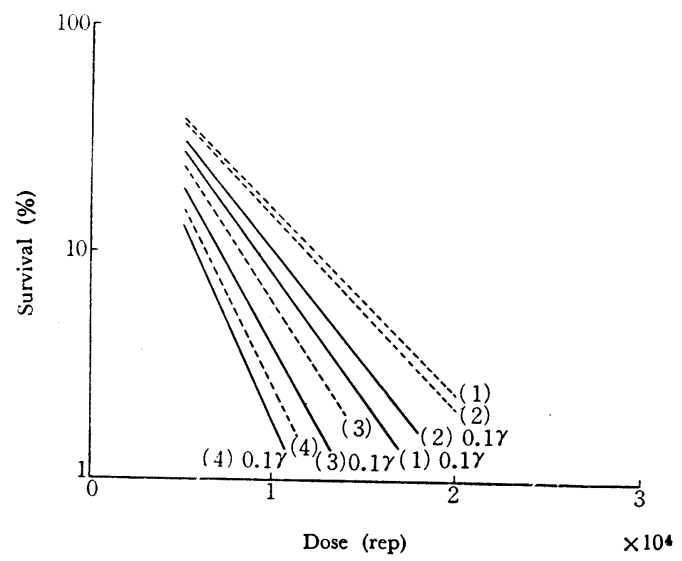

FIG. 8. Survival-curves of Bacteria Irradiated in Pure Agar Media Containing Achromycin.

(1) E. coli (2) A. aero. (3) P. vul. (4) Ps. aerug. ..... control
In the pure agar medium, the concentration tested was $0.1 \mathrm{mg} / \mathrm{ml}(\gamma)$ for all of the four organisms. The results are shown in Figure 8. These results show that the addition of Achromycin to the pure agar medium gave lower survival ratios with all of the strains.

\section{(5) Addition of Terramycin}

The concentrations of Terramycin added to the nutrient agar medium were $1 \mathrm{mcg} / \mathrm{ml}$ for

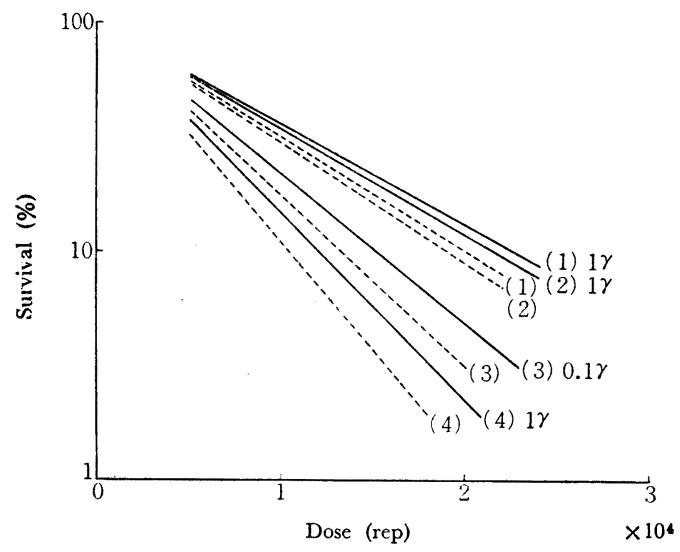

FIG. 9. Survival-curves of Bacteria Irradiated in Nutrient Media Containing Terramycin.

(1) E. coli (2) A. aero. (3) Pr. vul. (4) Ps. aerug. -.....-. control

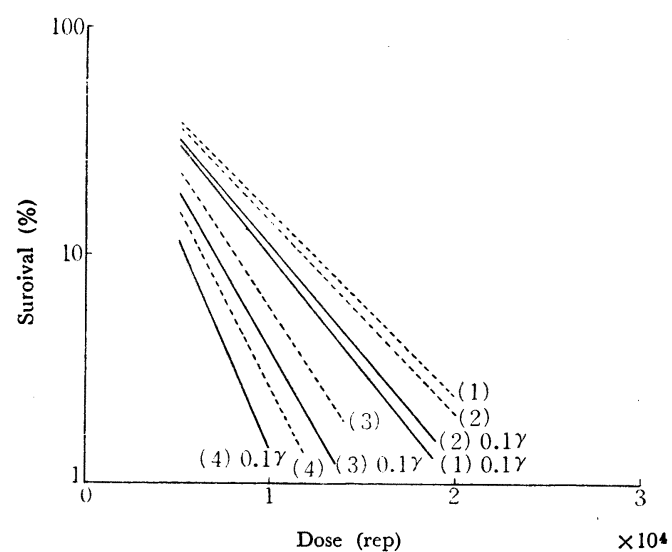

FIG. 10. Survival-curves of Bacteria Irradiated in Pure Agar Media Containitng Terramycin.

(1) E. coli (2) A. aero. (3) Pr. vul. (4) Ps. aerug. .....: control 
E. coli, A. aerogenes or Pseud. aeruginosa and $0.1 \mathrm{mcg} / \mathrm{ml}(\gamma)$ for Prot. vulgaris. The survival curves obtained are diagrammed in Figure 9. The pure agar medium was added with Terramycin in the same concentration of $0.1 \mathrm{mcg} / \mathrm{ml}(\gamma)$ for all the strains. The results are shown in Figure 10. As can be seen in these two figures, the addition of Terramycin to the nutrient agar medium resulted in higher survival ratios, while that to the pure agar medium in lower ratios. This tendency is the same as that observed with the other preservatives and antibiotics.

\section{DISCUSSION}

The experimental results show that the influences of the addition of preservative or antibiotic may differ depending upon the components of the medium. The addition of any of the agents tested to the nutrient agar medium resulted in higher survival ratios of the four species of bacteria, whereas, the addition to the non-nutritious pure agar medium resulted in lower survival ratios. These results are similar to those with thiourea, which had stronger influence when added to the nutrient medium. All of these substances are poisonous to the microorganisms tested, although the effectiveness may differ from one to another. Even so, a similar tendency was observed in the effect in combination with gamma-ray irradiation. It is not known, however, as to whether one-tenth of the maximum tolerating dose of these substances influenced the normal physiology of the bacteria when they were irradiated or whether the irradiation changed the nature of these agents in the nutrient agar medium.

\section{SUMMARY}

Sodium benzoate, dehydro acetic acid, Zfuran, tetracycline, or oxytetracycline was added to the nutrient agar medium or the pure agar medium at a concentration of onetenth of the maximum tolerating dose to study their effect upon the lethal effect of gamma-ray irradiation of four species of bacteria.

In the non-nutritious pure agar medium, these substances enhanced the lethal effect, whereas, in the nutrient agar medium these were rather protective.

Acknowledgement The author wishes to express his cordial thanks to Prof. T. Asai, Inst. Applied Microbiol., Univ. of Tokyo, and to Prof. T. Obara, Dept. of Agr. Chem., Tokyo Univ. of Education, for their invaluable advice and constant guidance. $\mathrm{He}$ is also indebted to Prof. D. Ushiba and Asist. Prof. S. Sasaki, Dept. of Bacteriology, Keio Univ., for their kind suggestions, and to Prof. H. Kadota, the Research Institute for Food Science, Kyoto University, for his helpful and valuable advice. Thanks are also due to Dr. H. Yamashita, the Radium Center of Cancer Institute, for his sound advice concerning radio-isotopes.

This study was supported in part by a Grantin-aid for Scientific Research defrayed from the Rockefeller Foundation. 